\title{
Fine Structures in Phase-Separated Domains of a Polyolefin Blend via Spinodal Decomposition
}

\author{
Xiaohua Zhang, ${ }^{\dagger}$ Zhigang Wang, ${ }^{\ddagger}$ Charles C. Han ${ }^{*}+$ \\ Beijing National Laboratory for Molecular Sciences, Joint Laboratory of Polymer \\ Science and Materials, State Key Laboratory of Polymer Physics and Chemistry, CAS \\ Key Laboratory of Engineering Plastics, Institute of Chemistry, Chinese Academy of \\ Sciences, Beijing 100080, China.
}

*To whom correspondence should be addressed. E-mail: c.c.han@iccas.ac.cn

\section{Supporting Information}

\section{Experimental Section}

The thermal behaviors of the blend were investigated using a differential scanning calorimeter (Mettler Toledo-822e) under nitrogen purge with a flux of ca. $50 \mathrm{~mL} / \mathrm{min}$ in order to prevent oxidative degradation of the samples during heating. Temperature calibration was performed with an indium standard. The film of the blend was obtained by compression molding at $160{ }^{\circ} \mathrm{C}$. Samples for DSC measurement were cut from the film and the weight was kept at ca. $3 \mathrm{mg}$. The cooling and heating rates were $10{ }^{\circ} \mathrm{C} / \mathrm{min}$. Different thermal treatments were applied to the blend samples prior to the DSC measurements. The samples were melted at $160{ }^{\circ} \mathrm{C}$ for $14 \mathrm{~min}$ to remove 
thermal history, then cooled to the selected LLPS temperature (Ts) of $135{ }^{\circ} \mathrm{C}$ for 13 , 16 and 24 h, respectively. Then, all the blend samples were quenched to room temperature before measurements.

\section{Result and Discussion Section}

Thermal behaviors associated with phase separation. DSC experiments were carried out further to illustrate the results obtained by OM and AFM associated to the second-step phase separation. Figure S1 shows the DSC results for PEH/PEB=30/70 blend after LLPS at $135{ }^{\circ} \mathrm{C}$ for different periods of time. The enthalpies of the higher temperature exothermal peak (peak 1) during cooling and the endothermal peak (peak 1) during subsequent heating decrease and those of the lower temperature ones (peak 2) become more obvious with the increasing time of LLPS. The explanation to the above phenomenon follows. As stated, either the higher branched PEH may concentrate in the PEB rich domains due to relatively higher solubility with PEB and forms imperfect lamellar crystals or the PEH concentration in the PEB rich domains is low and PEH crystallizes at lower temperature to form imperfect crystals. Therefore, peak 1 should mainly correspond to PEH crystals in PEH rich domains, while peak 2 should correspond to the PEH crystals in PEB rich domains. The temperature position of peak 1 shifts to lower crystallization temperature during cooling with the increasing time of LLPS. It could attribute to the slower nucleation rate of the blend undergoing LLPS for a long time, which provides weaker fluctuation assisted nucleation. ${ }^{1}$ In the same cooling rate of DSC, the sample with fast nucleation rate (less or no time for LLPS) can nucleation with a relatively higher crystallization temperature. 


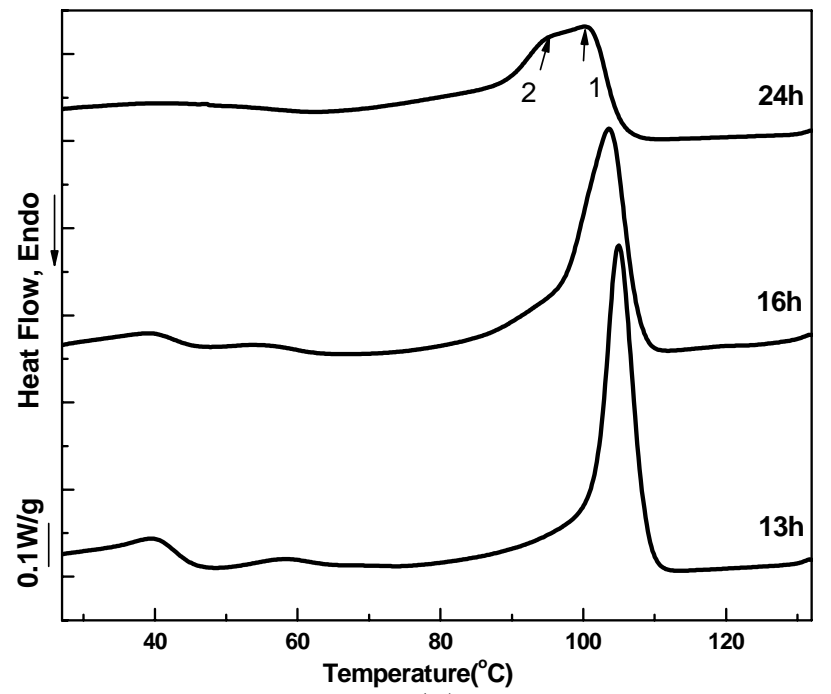

(a)

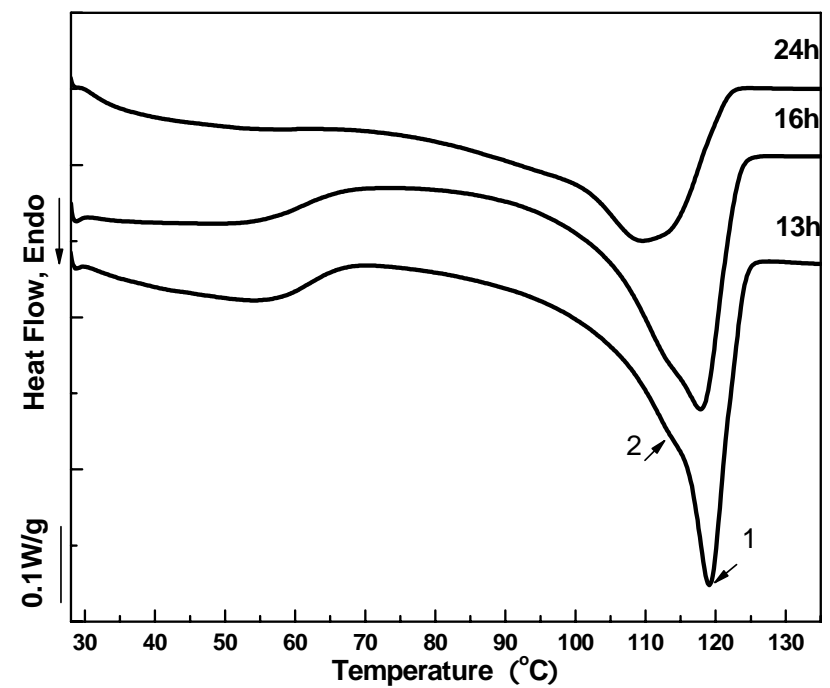

(b)

Figure S1. (a) Cooling DSC scans of PEH/PEB $=30 / 70$ blend after LLPS at $135^{\circ} \mathrm{C}$ for different times. (b) Subsequent heating DSC scans after the cooling runs shown in Figure S1a. 


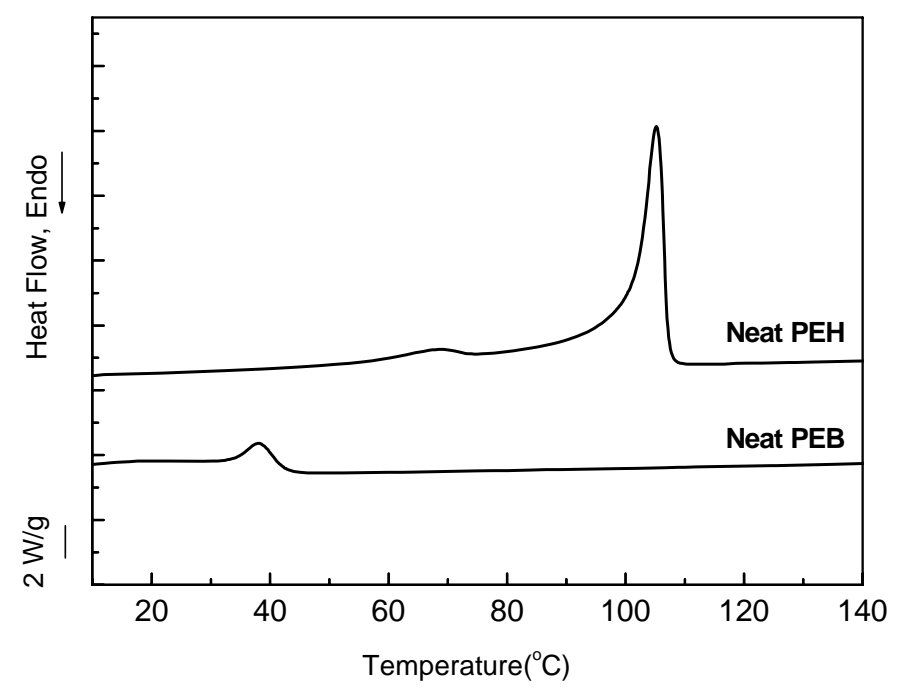

(a)

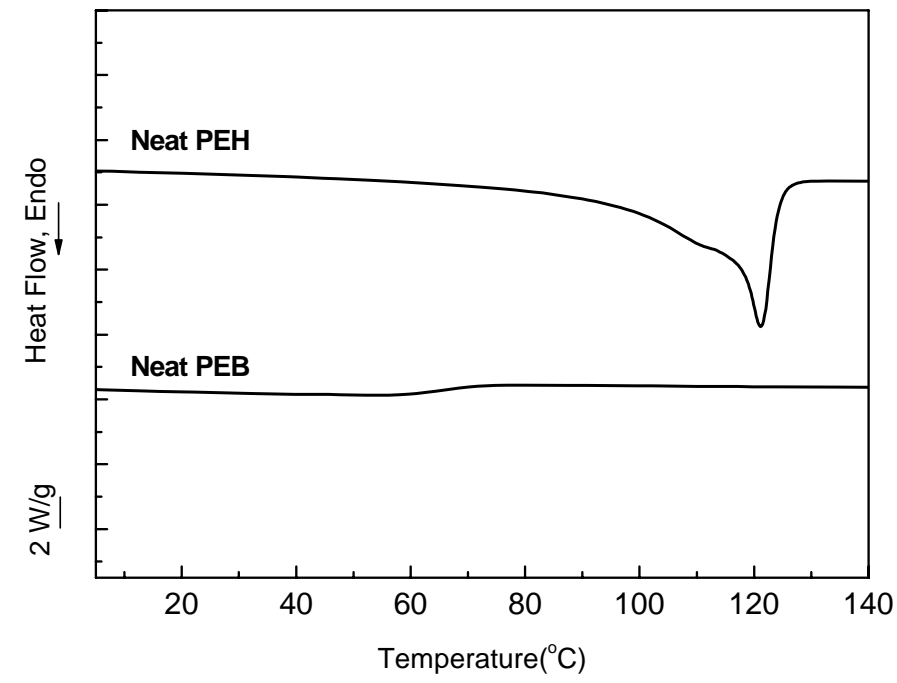

(b)

Figure S2. (a) Cooling DSC scans of neat PEH and PEB samples. (b) Subsequent heating DSC scans, after the cooling runs shown in Figure S2a.

\section{Reference Section}

(1) Zhang, X.; Wang, Z.; Dong, X.; Wang, D.; Han, C. C. J. Chem. Phys. 2006, 125, 024907. 